\title{
ALGUNAS ÁREAS DE TRABAJO ESENCIALES EN EL PROGRAMA DEL DIPLOMA DEL BACHILLERATO INTERNACIONAL
}

\section{SOME ESSENTIAL WORK AREAS IN THE INTERNATIONAL BACCALAUREATE DIPLOMA PROGRAM}

\begin{abstract}
Antonio Muñoz Castillo
\section{ABSTRACT}

The article presented is intended to offer a proposal for improvement in the implementation of the International Baccalaureate Diploma Program in certain areas that have been defined as essential for the development of the program. Three major areas of improvement are proposed for any school that offers the Diploma Program related to the following aspects: student academic performance, pedagogical leadership and teaching performance. These three lines of action cover the academic and organizational areas of schools and are essential to advance in the Diploma Program development. In addition, they are totally related to the standard and practices that rule the International Baccalaureate programs.

Consequently, the article places emphasis on some aspects that help the improvement of educational quality in schools in the framework and context of the implementation of the International Baccalaureate Diploma Program. In this way, the objective is to indicate specific areas of improvement and a series of associated good practices that can serve as an instrument in any IB World School to improve and further develop the Diploma Program.
\end{abstract}

Key words: International Baccalaureate, Diploma Program, Education, Leadership, Development plan

\section{RESUMEN}

El artículo que se presenta tiene como objetivo ofrecer una propuesta de mejora en la implementación del Programa del Diploma (PD) del Bachillerato Internacional en ciertas parcelas que se han definido como esenciales para el desarrollo del programa. De esta manera, se proponen tres grandes áreas de perfeccionamiento para cualquier centro educativo que imparta el PD relacionadas con los siguientes aspectos: rendimiento académico del alumnado, liderazgo pedagógico y desempeño docente. Estas tres líneas de actuación abarcan los ámbitos académico y organizativo de los centros educativos y son parcelas indispensables para hacer avanzar el Programa del Diploma, además, están totalmente relacionadas con la implementación de las normas y aplicaciones concretas que rigen los programas del Bachillerato Internacional.

Consecuentemente, este artículo pone énfasis en algunos aspectos que favorecen la mejora de la calidad educativa en el centro educativo en el marco y contexto de la implementación del PD del Bachillerato Internacional. De esta forma, el objetivo es señalar áreas concretas de trabajo y una serie de buenas prácticas asociadas que puedan servir de instrumento a cualquier colegio del Mundo IB para el perfeccionamiento y desarrollo del PD.

Palabras clave: Bachillerato Internacional, Programa del Diploma, Educación, Liderazgo, Plan de desarrollo

Fecha de recepción: 9 de octubre de 2020.

Fecha de aceptación: 1 de diciembre de 2020. 


\section{INTRODUCCIÓN}

Es evidente que todo proceso de administración de un centro educativo implica también la gestión directa de las aulas, $y$, por ende, tiene una repercusión directa en los procesos de enseñanza y aprendizaje. De esta forma, el proyecto de dirección de un colegio debe contemplar unos principios teóricos que orienten las acciones que se proponen. A su vez, ha de plantear objetivos alcanzables, realistas, claros y consensuados por toda la comunidad y debe estar adaptado a la realidad específica y las características concretas del centro ya que no existen recetas universales ni planes de mejora aplicables en todos los contextos. Como señalan Murillo y Krichesky (2000), cada proyecto debe ajustarse a las características del proceso, así como a sus destinatarios y finalidad.

No obstante, hay una serie de áreas de perfeccionamiento que se repiten en todos los Colegios del Mundo IB que ofrecen los programas del Bachillerato Internacional (IB) y que deben ser foco de todo plan de mejora orientado a impulsar el desarrollo de estos programas. En este sentido, no es suficiente que la escuela someta los programas a evaluaciones quinquenales por parte de la organización, sino que es necesario que el colegio identifique puntos fuertes y débiles y establezca áreas en las que centrar esfuerzos como forma de reforzar el desarrollo de los programas y asegurar la calidad educativa.

En este sentido, se ha tomado como base principal para este artículo las distintas normas y aplicaciones concretas que enmarcan los programas del IB, en este caso, del Programa del Diploma. Las normas constituyen condiciones generales que los colegios deben cumplir en la implementación de los programas y las aplicaciones concretas son la expresión práctica de las normas. Así, han sido particularmente relevantes ciertas normas y aplicaciones concretas que tienen una relación directa con las áreas que se proponen y sobre las que se centran los planes de actuación que se ofrecen.

Lógicamente, cada colegio, a partir de un diagnóstico basado en su contexto particular, puede vincular las necesidades detectadas con cambios programados que tengan en cuenta la realidad existente en el centro educativo y que conecten las líneas de actuación que se proponen aquí con lo que se viene haciendo en el colegio con el objetivo de impulsar líneas estratégicas adecuadas y planes de mejora en relación con la implementación del PD del Bachillerato Internacional.

En este aspecto, ya el propio Bachillerato Internacional recoge en el documento Normas para la implementación de los programas y aplicaciones concretas (2014) que "la implementación del programa es un proceso continuo y que las normas y aplicaciones se cumplirán en distintos grados a medida que se avanza en dicho proceso". Por lo tanto, se antoja esencial analizar también las dificultades que se detecten en referencia a la implementación de las distintas aplicaciones concretas desde la perspectiva de los profesores y el equipo de liderazgo como medio para progresar en el desarrollo del Programa del Diploma.

De esta manera, las propuestas de trabajo que se formulan en este artículo se ajustan perfectamente con la idea que se expresa en documentación del Bachillerato Internacional sobre la necesidad que tienen los Colegios del Mundo IB de mejorar continuamente la implementación de los programas y desarrollar un plan de acción que contenga pasos claros y acciones futuras con el objetivo de perfeccionar los procesos de enseñanza y aprendizaje. En concreto, la Guía para la evaluación del programa (2016) expresa que "se espera que los colegios traten de mejorar continuamente la calidad de la implementación de los programas mediante un compromiso continuado con el cumplimiento de todas las normas, aplicaciones y requisitos específicos del programa” (p.7). 
De este modo, la idea principal de este artículo es ofrecer áreas en las que centrar los esfuerzos para hacer avanzar el PD en parcelas fundamentales, evitando el estancamiento y permitiendo una mayor eficacia en la implementación de las normas y aplicaciones concretas que rigen los programas del Bachillerato Internacional. Las áreas de mejora que son foco en este trabajo son las siguientes: Rendimiento académico del alumnado, Liderazgo pedagógico y Desempeño docente.

Estas tres áreas de mejora abarcan los ámbitos académico y organizativo de los centros educativos ya que implican medidas de carácter pedagógico, pero también incluyen acciones relativas a la organización interna del colegio. Además, siguen la línea de lo indicado en numerosa literatura y documentación sobre qué aspectos definen a las buenas escuelas. Así, (Murillo, 2004, 2011) señala sobre la mejora de la eficacia escolar la importancia de tres tipos de objetivos en todo plan de mejora:

1. Objetivos dirigidos a la mejora del desarrollo de los estudiantes. Son los referidos a los resultados que se espera que obtengan los alumnos como fruto de su proceso de aprendizaje.

2. Objetivos dirigidos a incrementar la calidad de los centros en su conjunto. Preferentemente aquellos que la investigación ha encontrado que están asociados al desarrollo integral de los estudiantes. Son los que inciden en el funcionamiento general del centro y su impacto en el aprendizaje de los alumnos, en las características de liderazgo, las oportunidades de desarrollo profesional para el profesorado, el clima del centro, etc.

3. Objetivos de mejora de la calidad de los docentes individuales. Objetivos que han de convertirse en compromisos personales de mejora de cada uno de los docentes del centro educativo.

Así, los tres tipos de objetivos generales señalados por Murillo coinciden con las áreas marcadas:

-Rendimiento académico del alumnado (1-Objetivo dirigido a la mejora del desarrollo de los estudiantes y referidos a los resultados que se espera que obtengan los alumnos como fruto de su proceso de aprendizaje).

-Liderazgo pedagógico (2-Objetivo dirigido a incrementar la calidad de los centros en su conjunto).

-Desempeño docente (3-Objetivo de mejora de la calidad de los docentes individuales).

Estos objetivos se deben asociar a un plan de acción y tienen que contar con medidas específicas encaminadas a la mejora de estas parcelas, con identificación de la persona responsable, y con un cronograma de actuación para ello. Como parte de este plan de acción, también se debe ofrecer detalle sobre los recursos necesarios para llevarlo a cabo, tanto humanos como materiales, y los indicadores de resultados que se pretende alcanzar con la aplicación de cada acción prevista.

\section{RENDIMIENTO ACADÉMICO DEL ALUMNADO}

El rendimiento académico del alumnado está directamente relacionado con los procesos de enseñanza y aprendizaje que se llevan a cabo en el centro escolar y los resultados que obtienen los estudiantes están, de esta forma, conectados a la calidad de estos procesos. En consecuencia, el éxito o fracaso académico del alumno depende directamente de la efectividad de las prácticas que se lleven a cabo en el centro educativo. 
En este sentido, es numerosa la literatura que habla de que, sin aprendizaje, no hay resultados académicos buenos. Hargreaves y Fink (2006) comentan lo siguiente:

El cambio y la mejora sostenibles en la escuela comienzan con el propósito de alcanzar la integridad del resultado. Sitúan en primer lugar el aprendizaje, por delante del rendimiento escolar o de los exámenes. El aprendizaje se considera el requisito esencial para conseguir todo lo demás. El hecho de tener presente el aprendizaje hace que éste sea profundo y amplio, consiguiendo que vaya más allá de lo básico o fundamental (p. 49).

De esta manera, parece fundamental tener como base la frase de Hargreaves y Fink de que "el aprendizaje se considera el requisito esencial para conseguir todo lo demás" si se pretenden aumentar los resultados académicos en cualquier centro educativo, ya que, el camino para esta mejora reside, principalmente, en ajustar los procesos de enseñanza y aprendizaje del colegio.

El propio Bachillerato Internacional menciona en el documento El Programa del Diploma: de los principios a la práctica, (p. 23) la responsabilidad del equipo directivo del colegio de garantizar que los estándares en los procesos de enseñanza y aprendizaje son adecuados y mantienen un nivel de implementación alto. No obstante, también reconoce que los cambios en las prácticas de enseñanza requieren un importante apoyo por parte de todos los profesores y el personal de dirección y que es probable que el proceso sea lento y dificultoso, como ocurre siempre con los cambios que exigen examinar y modificar prácticas y modos de pensar. Sin embargo, también admite que el proceso de cambio tendrá un efecto positivo en todo el colegio, en cada profesor y, lo que es más importante, en la calidad del aprendizaje de los alumnos.

En consecuencia, es necesario plantear una serie de medidas en aspectos clave de la implementación del PD para ajustar los procesos de enseñanza y aprendizaje. Concretamente, es primordial considerar la metodología docente (enfoques de la enseñanza), el desarrollo de una serie de habilidades en los alumnos que les permita desarrollar con garantías de éxito las distintas tareas de evaluación interna y las pruebas externas (enfoques de la enseñanza) y los principios de la evaluación que rigen el programa.

De esta manera, se ha de buscar poner énfasis en un aprendizaje significativo y profundo a partir de una revisión de las prácticas y estrategias docentes para permitir que los alumnos desarrollen una serie de habilidades y competencias clave para aumentar su rendimiento académico. Además, es muy importante construir un andamiaje para el aprendizaje adecuado en el que se tenga en cuenta los conocimientos previos de los alumnos y en el que se consolide mediante actuaciones unas transiciones apropiadas entre el Programa de Años Intermedios (PAI) y el Programa del Diploma para asegurar una progresión satisfactoria de los estudiantes.

Por otra parte, y como medio para apoyar los procesos de enseñanza y aprendizaje, es también muy necesario determinar medidas enfocadas a realizar ajustes en cuestiones relacionadas con la evaluación y, en concreto, con la aplicación de los principios de la evaluación en el colegio para que reflejen los requisitos del PD. En esta línea, la evaluación es el puente entre la enseñanza y el aprendizaje y eso es verdaderamente lo importante, potenciar los principios de la evaluación como nexo entre metodología docente y aprendizaje perdurable de los estudiantes con el objetivo de aumentar la calidad de los resultados.

No obstante, un buen rendimiento académico del alumnado no debe ser un fin en sí mismo, sino una consecuencia de procesos de enseñanza y aprendizaje bien ajustados y de la aplicación de forma adecuada de los principios de evaluación que rigen los programas del Bachillerato Internacional. Es decir, sin una correcta planificación del aprendizaje los resultados de los alumnos no mejoran, 
consecuentemente, para buscar la mejora en el rendimiento académico del alumnado hay que potenciar aspectos básicos en lo que es la implementación del PD. En este sentido, se han determinado una serie de objetivos específicos asociados a distintas actuaciones y acciones como medio de progresar en esta parcela:

Tabla 1: Rendimiento académico del alumnado

\begin{tabular}{|c|c|}
\hline \multicolumn{2}{|c|}{ Objetivo general: Mejorar el rendimiento académico del alumnado en el PD } \\
\hline \multicolumn{2}{|c|}{$\begin{array}{l}\text { Objetivo específico 1: } \\
\text { Potenciar los principios de evaluación en el colegio para que reflejen los requisitos del PD }\end{array}$} \\
\hline Actuaciones & Acciones \\
\hline $\begin{array}{l}\text { Analizar los datos de la } \\
\text { evaluación para mejorar } \\
\text { la enseñanza y el } \\
\text { aprendizaje }\end{array}$ & $\begin{array}{l}\text {-Obtención de información específica y detallada relacionada con los } \\
\text { resultados de la evaluación interna y externa de las asignaturas y los } \\
\text { elementos troncales } \\
\text {-Uso de un sistema de análisis de los resultados de la evaluación } \\
\text { interna y externa de las asignaturas, los elementos troncales y los } \\
\text { datos relativos a logros pertinentes al programa, que permita } \\
\text { identificar puntos fuertes y débiles en áreas que necesitan mejoras en } \\
\text { la evaluación interna y externa y en el programa en general } \\
\text {-Desarrollo de intervenciones coordinadas basadas en el análisis de } \\
\text { los resultados de la evaluación interna y externa de las asignaturas, } \\
\text { los elementos troncales y los datos relativos a logros pertinentes al } \\
\text { programa que contribuyan a la calidad de la implementación del } \\
\text { programa y orienten el proceso de enseñanza y aprendizaje con el } \\
\text { objetivo de mejorar el funcionamiento, la sustentabilidad, la calidad } \\
\text { de la implementación y los resultados en el programa } \\
\text {-Medición del impacto de las intervenciones que surgen de los } \\
\text { análisis de la evaluación interna y externa de las asignaturas y los } \\
\text { elementos troncales }\end{array}$ \\
\hline $\begin{array}{l}\text { Utilizar una variedad de } \\
\text { estrategias y } \\
\text { herramientas para } \\
\text { evaluar el aprendizaje de } \\
\text { los alumnos }\end{array}$ & $\begin{array}{l}\text {-Promover un uso adecuado de una variedad de herramientas y } \\
\text { estrategias de evaluación en cada una de las áreas disciplinarias y } \\
\text { entre ellas para que los alumnos demuestren su aprendizaje } \\
\text {-Garantizar que la política de evaluación del colegio incluye: } \\
\text { - Un uso coherente y regular de la evaluación formativa y sumativa } \\
\text { - Un uso equilibrado de los diferentes tipos de estrategias de } \\
\text { evaluación: autoevaluación, evaluación entre compañeros... } \\
\text { - Una variedad de instrumentos de evaluación que incorporen } \\
\text { herramientas en línea y que permitan a los alumnos demostrar su } \\
\text { aprendizaje de diversas maneras y en distintos formatos } \\
\text {-Planificar, regularizar y sistematizar las prácticas de estandarización } \\
\text { interna de la evaluación en los distintos departamentos }\end{array}$ \\
\hline
\end{tabular}


Ofrecer información a los alumnos sobre su desempeño escolar para ayudarlos a mejorar
-Garantizar que la rutina en materia de evaluación incluye:

- Comentarios coherentes con la política de evaluación del colegio en materia de evaluación y la evaluación basada en criterios

- Comentarios formativos que los profesores hacen a los alumnos de manera regular por distintos medios para mejorar el aprendizaje de los alumnos

-Implementación de estrategias de manera sistemática para hacer un seguimiento del impacto de los comentarios formativos en el desempeño de los alumnos

-Uso eficaz de un sistema de registro del progreso de los alumnos

Tabla 2: Rendimiento académico del alumnado

Objetivo general: Mejorar el rendimiento académico del alumnado en el PD

Objetivo específico 2:

Potenciar la planificación y la reflexión en colaboración de los enfoques del aprendizaje para apoyar la implementación del PD

\begin{tabular}{|c|c|}
\hline Actuaciones & Acciones \\
\hline $\begin{array}{l}\text { Desarrollar actitudes y } \\
\text { habilidades que permitan } \\
\text { a los alumnos abordar } \\
\text { todos los objetivos } \\
\text { generales del Programa } \\
\text { del Diploma y los } \\
\text { específicos de cada } \\
\text { asignatura mediante un } \\
\text { diagrama de } \\
\text { planificación de las } \\
\text { habilidades de enfoques } \\
\text { del aprendizaje para cada } \\
\text { año del PAI y del PD }\end{array}$ & $\begin{array}{l}\text {-Programar reuniones de planificación y reflexión colaborativas de } \\
\text { manera periódica y regular, y de forma sistemática, donde se aborde } \\
\text { la articulación vertical y horizontal de las habilidades de enfoques del } \\
\text { aprendizaje y las habilidades comunes a las distintas disciplinas } \\
\text {-Aplicar un enfoque integrado del currículum que incluya a todas las } \\
\text { asignaturas para el desarrollo de habilidades específicas en los } \\
\text { alumnos } \\
\text {-Desarrollar un currículo escrito que especifique los conocimientos, } \\
\text { los conceptos, las habilidades y las actitudes que se van a desarrollar } \\
\text { con el tiempo } \\
\text {-Desarrollar aulas centradas en el desarrollo de habilidades } \\
\text { específicas en cada asignatura que conduzcan al éxito en las } \\
\text { evaluaciones del programa, tanto internas como externas }\end{array}$ \\
\hline $\begin{array}{l}\text { Basar la planificación y la } \\
\text { reflexión colaborativas } \\
\text { en expectativas de } \\
\text { aprendizaje } \\
\text { consensuadas que } \\
\text { garanticen que todos los } \\
\text { docentes tengan un } \\
\text { panorama general de las } \\
\text { experiencias educativas } \\
\text { de los alumnos }\end{array}$ & $\begin{array}{l}\text {-Garantizar que el currículo escrito toma como punto de partida las } \\
\text { experiencias de aprendizaje previas de los alumnos y tiene en cuenta } \\
\text { lo que los alumnos saben y pueden hacer } \\
\text {-Consensuar en reuniones de planificación y reflexión colaborativas } \\
\text { los objetivos del aprendizaje de forma clara y precisa } \\
\text {-Evaluar y monitorear el proceso de adquisición de habilidades por } \\
\text { parte de los estudiantes }\end{array}$ \\
\hline
\end{tabular}


Desarrollar actitudes y habilidades que permitan a los alumnos emprender acciones significativas en respuesta a sus propias necesidades de aprendizaje
-Garantizar la creación de un ambiente propicio para el aprendizaje de los alumnos

-Establecer un clima de relaciones de aceptación, equidad, confianza, solidaridad y respeto en el aula, manteniendo formas consistentes de convivencia en el aula

-Manifestar altas expectativas sobre las posibilidades de aprendizaje y desarrollo de todos los alumnos

-Establecer un ambiente organizado de trabajo y disponer los espacios y recursos en función de los distintos aprendizajes de los alumnos

-Asegurar que la enseñanza y el aprendizaje animan a los alumnos a reflexionar sobre cómo, qué y por qué aprenden

Fuente: Elaboración propia

Tabla 3: Rendimiento académico del alumnado

Objetivo general: Mejorar el rendimiento académico del alumnado en el PD

Objetivo específico 3:

Potenciar la planificación y la reflexión en colaboración de los enfoques de la enseñanza para apoyar la implementación del PD

\begin{tabular}{|c|c|}
\hline Actuaciones & Acciones \\
\hline $\begin{array}{lcr}\text { Asegurar } & \text { que } & \text { la } \\
\text { enseñanza } & \text { y } & \text { el } \\
\text { aprendizaje } & \text { animan } & \text { los } \\
\text { alumnos } & \text { a } & \text { ser } \\
\text { indagadores } & & y \\
\text { pensadores } & & \end{array}$ & $\begin{array}{l}\text {-Usar la indagación como metodología docente en todas las } \\
\text { asignaturas, con énfasis en lo siguiente: } \\
\text { - Desarrollo de habilidades de pensamiento } \\
\text { - La agencia de los alumnos } \\
\text { - La integración de Teoría del Conocimiento en la enseñanza } \\
\text { de todas las asignaturas del Programa del Diploma } \\
\text {-Elaborar planes de unidades donde se potencien estrategias de } \\
\text { enseñanza y aprendizaje que fomenten el pensamiento crítico, la } \\
\text { indagación y la reflexión } \\
\text {-Incorporar metodologías de enseñanza-aprendizaje innovadoras } \\
\text { (Aprendizaje Orientado a Proyectos; Aprendizaje basado en } \\
\text { Problemas; Investigación-Acción; Aprendizaje Cooperativo...) en } \\
\text { los planificadores de unidad } \\
\text {-Proporcionar formación al profesorado en metodologías de } \\
\text { Enseñanza-Aprendizaje innovadoras } \\
\text {-Establecer un plan de observaciones que garantice que la enseñanza } \\
\text { y el aprendizaje animan a los alumnos a ser indagadores y pensadores }\end{array}$ \\
\hline
\end{tabular}




\begin{tabular}{|c|c|}
\hline $\begin{array}{l}\text { Garantizar que la } \\
\text { enseñanza y el } \\
\text { aprendizaje utilizan una } \\
\text { variedad de estrategias } \\
\text { metodológicas }\end{array}$ & $\begin{array}{l}\text {-Elaborar planes de unidades donde se use una variedad de } \\
\text { estrategias y metodologías docentes } \\
\text {-Incorporar metodologías de enseñanza-aprendizaje innovadoras: } \\
\text { (Aprendizaje Orientado a Proyectos; Aprendizaje basado en } \\
\text { Problemas; Investigación-Acción; Aprendizaje Cooperativo...) en } \\
\text { los planificadores de unidad } \\
\text {-Fomentar y utilizar las tecnologías de la información como apoyo a } \\
\text { metodologías docentes innovadoras } \\
\text {-Formación del profesorado en metodologías de Enseñanza- } \\
\text { Aprendizaje innovadoras } \\
\text {-Garantizar que la enseñanza y el aprendizaje aborden diferentes } \\
\text { estilos de aprendizaje mediante una variedad de prácticas } \\
\text { pedagógicas } \\
\text {-Establecer un plan de observaciones que garantice que la enseñanza } \\
\text { y el aprendizaje utilizan una variedad de estrategias metodológicas }\end{array}$ \\
\hline $\begin{array}{l}\text { Utilizar estrategias de } \\
\text { diferenciación con el fin } \\
\text { de cubrir las necesidades } \\
\text { y estilos de aprendizaje } \\
\text { de todos los alumnos }\end{array}$ & $\begin{array}{l}\text {-Usar una mayor variedad de estrategias metodológicas para atender } \\
\text { necesidades y estilos de aprendizaje diferentes } \\
\text {-Fomentar y utilizar las tecnologías de la información como recurso } \\
\text { para apoyar la diferenciación } \\
\text {-Garantizar que la enseñanza y el aprendizaje abordan los diferentes } \\
\text { estilos de aprendizaje y necesidades de los alumnos } \\
\text {-Establecer un plan de observaciones que garantice que la enseñanza } \\
\text { y el aprendizaje cubre las necesidades y estilos de aprendizaje de } \\
\text { todos los alumnos } \\
\text {-Asegurar mediante pruebas diagnósticas de distinto tipo que los } \\
\text { profesores conocen las necesidades y estilos de aprendizaje de los } \\
\text { alumnos de su clase } \\
\text {-Potenciar el uso de la diferenciación (por tarea, resultado o } \\
\text { agrupación) para que constituya una parte integral de la práctica } \\
\text { docente } \\
\text {-Garantizar que la práctica docente es coherente con la política de } \\
\text { inclusión o de necesidades educativas especiales del colegio } \\
\text {-Fomentar que el personal especializado facilite el acceso al } \\
\text { aprendizaje, cuando corresponda (por ejemplo, proporciona apoyo } \\
\text { en clase y durante la separación de los alumnos con necesidades de } \\
\text { apoyo educativo) }\end{array}$ \\
\hline
\end{tabular}

Fuente: Elaboración propia 


\section{LIDERAZGO PEDAGÓGICO}

Evidentemente, como parte de la labor de todo líder en un colegio, está la idea de definir el futuro de la institución educativa a largo plazo y crear una visión compartida de los principios y prácticas de los programas educativos presentes en el centro. Además, está la exigencia de intentar alcanzar un compromiso personal por parte de toda la comunidad para apoyar plenamente dichos principios y prácticas y permitir un desarrollo continuo de tales programas.

Asimismo, es evidente que en todo colegio es necesario crear condiciones para la enseñanza y el aprendizaje y que ese aprendizaje debe estar dirigido y organizado mediante prácticas profesionales adecuadas. De esta forma, y como bien señala Murillo (2006), las cuestiones pedagógicas son las más significativas en la escuela y, además, determinantes para lograr una enseñanza de calidad que permita a los estudiantes alcanzar su máximo potencial.

En este sentido, los programas del Bachillerato Internacional exigen que, tanto el director como el coordinador, ejerzan un liderazgo pedagógico en el colegio ya que tienen la responsabilidad de asegurar la mejora de la enseñanza y el aprendizaje mediante la aplicación de la filosofía y el cumplimiento de los requisitos de implementación del programa, según lo establecido en Normas para la implementación de los programas y aplicaciones concretas (2014).

De esta forma, la propia Organización del Bachillerato Internacional señala en el documento Cómo hacer realidad el PEP. Liderazgo pedagógico en los colegios (p.18) las siguientes líneas de actuación en referencia al liderazgo pedagógico:

- Fomentar un modelo de liderazgo compartido que requiera colaboración dentro del equipo responsable

- Programar tiempo para dedicarlo a la colaboración eficaz en todo el colegio

- Fortalecer la comunicación entre los distintos miembros de la comunidad escolar

- Desarrollar procesos de contratación eficaz que tengan en cuenta las necesidades del programa

- Usar eficaz y frecuentemente métodos para recibir comentarios y opiniones por parte de toda la comunidad escolar

- Empoderar a los docentes para que puedan tomar decisiones relativas al desarrollo y la implementación del programa

- Crear un clima que permita a los alumnos y docentes ser miembros autónomos de comunidades de aprendizaje

- Apoyar la reflexión y el cambio constantes y a largo plazo en la comunidad escolar

- Establecer objetivos relativos a la implementación del programa que sean concretos, que se puedan lograr y se ajusten a un calendario

- Proporcionar oportunidades de desarrollo profesional diferenciado al equipo docente como forma de apoyar y enriquecer el programa

- Ampliar las oportunidades para un desarrollo profesional continuo

En otro documento fundamental de referencia para la implementación del PD, El Programa del Diploma: de los principios a la práctica, se enfatiza la importancia del liderazgo pedagógico compartido, señalando lo siguiente:

El coordinador del PD cumple una función de liderazgo fundamental en la implementación y el desarrollo del programa. Es importante que la descripción de su puesto incluya dicha función de 
liderazgo, además de la gestión de las tareas de administración general del programa y de comunicación. La implementación del PD requiere amplia experiencia y conocimientos en cuanto a gestión de cambios, y una comprensión cabal de los principios y prácticas del programa. El coordinador, juntamente con otros integrantes del equipo directivo, debe tener la autoridad necesaria para planificar y administrar los procesos de cambio necesarios para desarrollar el programa (p.26).

Consecuentemente, se antoja absolutamente vital en procesos de mejora la labor que pueda desarrollar el equipo de liderazgo pedagógico de un colegio ya que es primordial para que el compromiso con los cambios se asuma por parte de todos los miembros de la comunidad y se trabaje en pos de ellos. Además, y como mencionan los documentos de posición del Bachillerato Internacional, en concreto, Cómo hacer realidad el PEP. Liderazgo pedagógico en los colegios (p.25) el equipo de liderazgo pedagógico debe tener la exigencia de depurar constantemente la implementación del programa y no permitir su estancamiento, proyectando hacia el futuro una visión compartida en la comunidad escolar:

El equipo de liderazgo pedagógico del colegio debe apreciar que su responsabilidad va más allá de mantener el funcionamiento del programa en el colegio. A largo plazo, su responsabilidad es generar una comprensión profunda del programa y lograr que tenga un impacto en todo el colegio para que la comunidad lo implemente con mayor confianza y perciba cómo prospera.

De esto se desprende que, y no solo en Colegios del Mundo IB el equipo directivo de los centros educativos son los principales agentes del cambio para la mejora al ser los responsables de establecer e impulsar planes que tengan como meta el progreso en la implementación de los programas educativos.

Consecuentemente, el trabajo en equipo y la gestión de los equipos educativos existentes en el centro es un elemento esencial. Así, se proponen medidas encaminadas a potenciar el liderazgo pedagógico en el centro como medio para perfeccionar el desarrollo del Programa del Diploma y alcanzar la excelencia educativa, garantizando que los estándares en la implementación del programa se mantienen altos. De esta forma, el objetivo principal es fortalecer el liderazgo pedagógico del equipo directivo del colegio para apoyar la implementación y desarrollo del PD. Como manera de lograr esta meta se han señalado una serie de objetivos específicos en referencia al principal para permitir su consecución:

Tabla 4: Liderazgo pedagógico

Objetivo general: Fortalecer el liderazgo pedagógico del equipo directivo para apoyar la implementación del PD

Objetivo específico 1:

Desarrollar, coordinar y supervisar el desarrollo del currículum, garantizando un progreso constante en la implementación del PD y una mejora continua de los procesos de enseñanza y aprendizaje en el colegio por parte del equipo de liderazgo.

Actuaciones

Acciones 


\begin{tabular}{|c|c|}
\hline $\begin{array}{l}\text { Brindar orientación y } \\
\text { fomentar el progreso } \\
\text { pedagógico de los } \\
\text { docentes en la enseñanza } \\
\text { de sus asignaturas por } \\
\text { parte del equipo de } \\
\text { liderazgo }\end{array}$ & $\begin{array}{l}\text {-Planificar, liderar y sistematizar reuniones de planificación } \\
\text { colaborativa } \\
\text { para respaldar a los profesores en la implementación del programa. } \\
\text {-Ofrecer apoyo y asistencia continua y regular en la implementación } \\
\text { del programa }\end{array}$ \\
\hline $\begin{array}{l}\text { Ofrecer orientación en } \\
\text { relación a la } \\
\text { implementación de las } \\
\text { normas y aplicaciones } \\
\text { concretas que rigen el } \\
\text { programa por parte del } \\
\text { equipo de liderazgo }\end{array}$ & $\begin{array}{l}\text {-Coordinar la implementación de los siguientes componentes del } \\
\text { programa: } \\
\text { - Enfoques de la enseñanza } \\
\text { - Buenas prácticas docentes basadas en indagación, pensamiento } \\
\text { crítico y reflexión } \\
\text {-Comprensión conceptual } \\
\text {-Enseñanza diferenciada } \\
\text { - Enfoques del aprendizaje } \\
\text { - Integración de los componentes troncales } \\
\text { - Planificación colaborativa } \\
\text { - Prácticas de evaluación } \\
\text { - Desarrollo de la mentalidad internacional } \\
\text { - Desarrollo de los atributos del perfil de la comunidad de } \\
\text { aprendizaje }\end{array}$ \\
\hline $\begin{array}{l}\text { Planificar las } \\
\text { expectativas de } \\
\text { aprendizaje previas al } \\
\text { Programa del Diploma } \\
\text { por parte del equipo de } \\
\text { liderazgo }\end{array}$ & $\begin{array}{l}\text {-Coordinar la transición entre programas (PAI y PD) } \\
\text {-Coordinar la elaboración de una planificación vertical de enfoques } \\
\text { del aprendizaje que cubra el PAI y el PD }\end{array}$ \\
\hline $\begin{array}{c}\text { Supervisar } \\
\text { la práctica docente y los } \\
\text { resultados académicos } \\
\text { que se vayan logrando } \\
\text { por parte del equipo de } \\
\text { liderazgo }\end{array}$ & $\begin{array}{l}\text {-Planificar el desarrollo profesional de los docentes } \\
\text {-Planificar y desarrollar la capacitación inicial para docentes nuevos } \\
\text {-Coordinar la evaluación del desempeño docente } \\
\text {-Recoger y analizar los datos de resultados de la evaluación interna } \\
\text { y externa }\end{array}$ \\
\hline
\end{tabular}

Fuente: Elaboración propia

Tabla 5: Liderazgo pedagógico

Objetivo general: Fortalecer el liderazgo pedagógico del equipo directivo para apoyar la implementación del PD

Objetivo específico 2:

Fomentar prácticas de liderazgo pedagógico a través de la interacción y la colaboración en el colegio 


\begin{tabular}{|c|c|}
\hline Actuaciones & Acciones \\
\hline $\begin{array}{l}\text { Aumentar el trabajo en } \\
\text { colaboración entre el } \\
\text { equipo de liderazgo } \\
\text { pedagógico y los } \\
\text { profesores del PD en } \\
\text { referencia a la } \\
\text { planificación, la } \\
\text { enseñanza y la } \\
\text { evaluación en equipo }\end{array}$ & $\begin{array}{l}\text {-Regularizar y sistematizar las reuniones de planificación } \\
\text { colaborativa } \\
\text {-Programar reuniones de planificación y reflexión colaborativas de } \\
\text { manera periódica donde se aborde la planificación, la enseñanza y la } \\
\text { evaluación en equipo del programa }\end{array}$ \\
\hline $\begin{array}{l}\text { Desarrollar e } \\
\text { implementar en } \\
\text { colaboración políticas, } \\
\text { sistemas y } \\
\text { procedimientos para } \\
\text { mejorar el } \\
\text { funcionamiento del } \\
\text { programa }\end{array}$ & $\begin{array}{l}\text {-Revisar las políticas existentes en el colegio (admisión, lingüística, } \\
\text { honestidad académica, necesidades educativas especiales y } \\
\text { evaluación) para asegurar que respalden adecuadamente la } \\
\text { implementación del programa y que estén integradas en la vida } \\
\text { escolar } \\
\text {-Desarrollar sistemas y protocolos que garanticen la continuidad y } \\
\text { el desarrollo del programa } \\
\text {-Actualizar el plan de acción del colegio para que sirva de } \\
\text { documento de ruta al colegio en la implementación del programa }\end{array}$ \\
\hline $\begin{array}{l}\text { Contribuir a generar una } \\
\text { cultura de evaluación } \\
\text { para la mejora en todo el } \\
\text { colegio que garantice la } \\
\text { continuidad y la mejora } \\
\text { progresiva del desarrollo } \\
\text { del programa }\end{array}$ & $\begin{array}{l}\text {-Fomentar la reflexión, la autoevaluación y la evaluación como } \\
\text { medio para determinar áreas de mejora por distintos medios } \\
\text { (reuniones, cuestionarios, grupos de discusión, análisis de } \\
\text { documentación...) y poder actuar entonces en acciones específicas }\end{array}$ \\
\hline
\end{tabular}

Fuente: Elaboración propia

Tabla 6: Liderazgo pedagógico

Objetivo general: Fortalecer el liderazgo pedagógico del equipo directivo para apoyar la implementación del PD

Objetivo específico 3:

Crear y organizar estructuras que faciliten la coordinación y el desarrollo del PD y el trabajo en equipo

Actuaciones

Acciones 


\begin{tabular}{|c|c|}
\hline $\begin{array}{l}\text { Determinar una } \\
\text { estructura de apoyo a la } \\
\text { implementación del } \\
\text { programa: coordinador } \\
\text { Monografía, coordinador } \\
\text { Enfoques de aprendizaje, } \\
\text { coordinador Enfoques } \\
\text { de la enseñanza y } \\
\text { coordinador CAS }\end{array}$ & $\begin{array}{l}\text {-Distribuir el liderazgo pedagógico en el colegio con el } \\
\text { nombramiento de distintas personas con cargos relacionados con } \\
\text { diferentes aspectos del PD } \\
\text {-Coordinar planes de implementación de prácticas concretas entre } \\
\text { los miembros de la estructura de apoyo al PD }\end{array}$ \\
\hline $\begin{array}{l}\text { Definir } \\
\text { responsabilidades de } \\
\text { cada miembro de la } \\
\text { estructura de apoyo a la } \\
\text { implementación del } \\
\text { programa }\end{array}$ & $\begin{array}{l}\text {-Elaborar una descripción de los puestos pertenecientes a la } \\
\text { estructura de apoyo al PD que incluya responsabilidades y } \\
\text { obligaciones en referencia a los mismos }\end{array}$ \\
\hline $\begin{array}{l}\text { Facultar a quienes } \\
\text { desempeñan cargos en la } \\
\text { estructura de apoyo a la } \\
\text { implementación del } \\
\text { programa para que } \\
\text { puedan cumplir con sus } \\
\text { responsabilidades y } \\
\text { liderar acciones } \\
\text { relacionadas con su rol }\end{array}$ & $\begin{array}{l}\text {-Apoyar públicamente a las personas que pertenecen a la estructura } \\
\text { de apoyo a la implementación del PD en referencia a la función que } \\
\text { desempeñan y resaltar y enfatizar sus roles y responsabilidades }\end{array}$ \\
\hline
\end{tabular}

Fuente: Elaboración propia

\section{DESEMPEÑO DOCENTE}

Según Guarro (2013), las buenas escuelas se caracterizan por un desarrollo profesional del profesorado comprometido con la mejora de las prácticas docentes, centrado en los problemas concretos y más habituales que generan las prácticas docentes con la intención de mejorarlas. De esta forma, parece evidente la permanente necesidad de mejora de la acción docente y la conexión entre eficacia en el desempeño pedagógico y aprendizaje de los alumnos.

Por otra parte, y como señalan numerosos estudios, es necesaria la evaluación y la autoevaluación de la práctica docente para que haya mejora y progreso. La propia organización del Bachillerato Internacional señala lo siguiente en el documento Cómo hacer realidad el PEP. Liderazgo pedagógico en los colegios (p.15):

El método empleado depende de cada colegio, pero siempre debe formar parte del proceso de evaluación de la pertinencia y eficacia del programa. Debe servir de apoyo a las prácticas reflexivas en todo el colegio y promover una actitud de aprendizaje activo durante toda la vida. Tanto la persona responsable de la evaluación (generalmente, el director) y los profesores o el coordinador del Programa deben buscar indicios del empleo de prácticas adecuadas basándose en la descripción de funciones del puesto pertinente.

Consecuentemente, reflexionar sobre cómo se enseña es absolutamente indispensable para identificar fortalezas y debilidades, pero esto no basta para alcanzar la excelencia, es necesario también establecer protocolos que permitan, mediante actuaciones de observación del docente, 
que puedan ser descritas y evaluadas, y análisis de resultados y documentación pertinente, determinar la competencia del profesor y su nivel de comprensión de la filosofía y las normas y aplicaciones concretas que marcan la implementación del Programa del Diploma.

Además de la evaluación del desempeño docente, es esencial que se fortalezca el desarrollo profesional de los profesores mediante distintas acciones que promuevan el aprendizaje en el profesorado. Este objetivo es señalado en el propio perfil de la comunidad de aprendizaje del IB, donde se menciona el valor de desarrollar una actitud activa de aprendizaje durante toda la vida. Además, son numerosos los estudios que manifiestan la necesidad de potenciar el desarrollo profesional de los docentes en procesos de cambio escolar.

Así, por ejemplo, Ávalos (2011), menciona que, en la medida que los profesores aprenden, adquieren el poder necesario para ejercer un liderazgo docente dirigido a transformaciones en la organización escolar. De esta forma, parece deseable vincular el desarrollo profesional de los docentes a la evaluación del desempeño individual de los profesores cuando se hayan detectado carencias en estas valoraciones.

No obstante, el Bachillerato Internacional, en todos sus programas, exige, independientemente de la evaluación del desempeño que pueda hacer el propio centro, que los profesores reciban capacitación oficial IB si el docente no la recibió en otra escuela. En consecuencia, es obligatorio para los colegios coordinar acciones de formación cuando se incorpora un docente a impartir clase en el PD. De hecho, el documento de Normas para la implementación de los programas y aplicaciones concretas en una de las aplicaciones concretas (B2.3) menciona lo siguiente: "El colegio se asegura de que sus profesores y el personal de dirección reciban desarrollo profesional aprobado por el IB" (p.22).

En este sentido, y para que el colegio lleve a cabo un plan de desarrollo profesional sostenido a largo plazo, hace falta, además de planificación, disponer recursos económicos y de tiempo para ello. Por lo tanto, es esencial que se destinen medios de forma regular para que se puedan realizar las acciones de formación que se consideren necesarias en el colegio o que sean requeridas por la organización del Bachillerato Internacional.

En la misma línea de apoyo al docente nuevo al centro comentada, es realmente importante que, además de llevar a cabo el desarrollo profesional externo obligado, se favorezca su integración en el colegio y la comprensión del PD y sus requerimientos mediante distintas acciones que garanticen que el profesor está en condiciones de realizar los procesos de enseñanza y aprendizaje con garantías.

Entre estas acciones, y no exclusivamente ya para docentes nuevos, sino para todo el claustro de profesores, es fundamental el compromiso con el aprendizaje y la mejora profesional de los docentes como parte del desarrollo profesional. Así, generar y sustentar culturas organizativas que tengan como fin la creación y participación en comunidades de aprendizaje debe ser parte del desarrollo profesional de los profesores para que impacte positivamente en el desempeño profesional docente y, por consiguiente, en los procesos de enseñanza y aprendizaje.

Gairín y Rodríguez-Gómez (2011) hablan de la importancia para el desarrollo personal y profesional de los docentes de estas comunidades de aprendizaje y de las posibilidades que se ofrecen para ello: crear y utilizar un banco de experiencias, promover grupos de creación de conocimiento sobre retos profesionales concretos, difundir y debatir proyectos, promover 
intercambios con otras instituciones, crear redes para el desarrollo de ideas, dar sentido al compromiso con la mejora de la organización y entorno, etc.

En consecuencia, se han determinado medidas que puedan ayudar a determinar debilidades en la actuación de los docentes con el objetivo de corregirlas y acciones que pueden fortalecer el desempeño profesional de los profesores, tanto de los profesores nuevos en el colegio como de los profesores con más experiencia. En esta área de desarrollo es básico que el centro educativo cree planes individuales de desarrollo profesional del profesorado que permitan cubrir las necesidades detectadas.

Tabla 7: Desempeño docente

Objetivo general: Mejorar el desempeño docente para apoyar la implementación del PD

Objetivo específico 1:

Evaluar el nivel de desempeño de los profesores

\begin{tabular}{|l|l|}
\hline Actuaciones & \multicolumn{1}{|c|}{ Acciones } \\
\hline $\begin{array}{l}\text { Determinar un plan de } \\
\text { observaciones, tanto } \\
\text { formales como } \\
\text { informales, que valore el } \\
\text { desempeño docente y un } \\
\text { marco para ello }\end{array}$ & $\begin{array}{l}\text {-Definir qué se va a observar en las visitas a las clases } \\
\text { la observación } \\
\text {-Planificar un calendario de observaciones que incluya fechas y } \\
\text { responsables de las observaciones } \\
\text {-Identificar fortalezas y áreas de mejora tras la observación } \\
\text {-Proporcionar retroalimentación al profesor sobre su actuación con } \\
\text { el objetivo de favorecer la reflexión sobre la práctica docente } \\
\text {-Establecer un plan de acción individualizado para el profesor } \\
\text { focalizado en las áreas de mejora } \\
\text {-Disponer medidas de seguimiento del plan de acción del profesor }\end{array}$ \\
\hline $\begin{array}{l}\text { Analizar datos objetivos } \\
\text { provenientes de los } \\
\text { resultados académicos de } \\
\text { alumnos ye los } \\
\text { informes de evaluación } \\
\text { interna }\end{array}$ & $\begin{array}{l}\text {-Obtener información sobre las prácticas de instrucción y una } \\
\text { comprensión profunda de los componentes y resultados de la } \\
\text { evaluación } \\
\text {-Identificar fortalezas y áreas de mejora para determinar } \\
\text { intervenciones según sea necesario } \\
\text {-Determinar necesidades de desarrollo profesional en el profesorado } \\
\text { a partir del análisis de resultados } \\
\text {-Establecer mecanismos y protocolos para compartir datos con el } \\
\text { personal docente para asegurar que se identifiquen las necesidades } \\
\text { de apoyo de los estudiantes } \\
\text {-Analizar información holística sobre el desarrollo académico del PD } \\
\text { en el colegio con el fin de valorar el plan de estudios del colegio } \\
\text {-Asegurar que se abordan las necesidades de aprendizaje de los } \\
\text { estudiantes a partir del análisis de los datos y se toman decisiones } \\
\text { académicas adecuadas (por ejemplo, la elección de idiomas en las } \\
\text { asignaturas de los grupos 1 y 2) } \\
\text {-Determinar necesidades de recursos y materiales en las distintas } \\
\text { asignaturas a partir del análisis de los resultados }\end{array}$ \\
\hline
\end{tabular}


Analizar la información sobre el desempeño docente proveniente de diversas instancias: alumnos, exalumnos, familias, compañeros de departamento, jefe departamento, coordinador del PD

-Determinar medios e instrumentos para recabar información sobre el desempeño de los docentes en función de diversos factores: preparación de las clases, didáctica, metodología, comunicación, interés en el estudiante, entusiasmo, respeto por los alumnos, responsabilidades profesionales...

-Establecer un calendario para la obtención de la información sobre el desempeño de los docentes, idealmente a mitad de curso y final del año académico

-Determinar un sistema de revisión del trabajo de los alumnos en cuadernos, portafolios, plataformas digitales y de las pruebas de evaluación aplicadas, tanto formativas como sumativas

-Hacer un seguimiento de los objetivos de aprendizaje alcanzados por los alumnos en las distintas materias

-Implantar un sistema de revisión por pares del trabajo del docente por uno o más de sus propios compañeros

\section{Fuente: Elaboración propia}

Tabla 8: Desempeño docente

\begin{tabular}{|c|c|}
\hline \multicolumn{2}{|c|}{$\begin{array}{l}\text { Objetivo específico } 2 \text { : } \\
\text { Generar y sustentar culturas organizativas que adopten un compromiso con el aprendizaje y la } \\
\text { mejora profesional }\end{array}$} \\
\hline Actuaciones & Acciones \\
\hline $\begin{array}{lr}\text { Promover } & \text { la } \\
\text { participación y } & \text { el } \\
\text { sostenimiento } & \text { de } \\
\text { comunidades } & \text { de } \\
\text { aprendizaje, presenciales } \\
\text { y en red, entre los } \\
\text { distintos miembros de la } \\
\text { comunidad escolar } \\
\end{array}$ & $\begin{array}{l}\text {-Fomentar la participación en organizaciones colaborativas que } \\
\text { generen confianza, relaciones positivas y un entorno laboral propicio } \\
\text { para la colaboración y el intercambio de conocimiento entre los } \\
\text { profesores } \\
\text {-Favorecer el aprendizaje entre los distintos grupos de la comunidad } \\
\text { escolar a través de compartir conocimiento, experiencia y experticia } \\
\text { y co-construir aprendizaje y significado entre ellos }\end{array}$ \\
\hline $\begin{array}{l}\text { Promover una actitud de } \\
\text { aprendizaje activo } \\
\text { durante toda la vida }\end{array}$ & $\begin{array}{l}\text {-Organizar y promover intercambios de buenas prácticas docentes, } \\
\text { internamente y con otros centros educativos } \\
\text {-Favorecer la indagación profesional y el aprendizaje colaborativo } \\
\text { sobre diversos temas basado en la acción o en la experiencia }\end{array}$ \\
\hline
\end{tabular}

Fuente: Elaboración propia 
Tabla 9: Desempeño docente

\begin{tabular}{|c|c|}
\hline \multicolumn{2}{|r|}{$\begin{array}{l}\text { Objetivo específico 3: } \\
\text { Potenciar el desarrollo profesional de los profesores }\end{array}$} \\
\hline Actuaciones & Acciones \\
\hline $\begin{array}{l}\text { Elaborar planes de } \\
\text { desarrollo profesional } \\
\text { para los docentes y } \\
\text { designar un presupuesto } \\
\text { para ello }\end{array}$ & $\begin{array}{l}\text {-Planificar actividades de aprendizaje para todos los docentes y } \\
\text { promover oportunidades de desarrollo profesional de común } \\
\text { acuerdo } \\
\text {-Determinar necesidades específicas de formación, actualización y } \\
\text { desarrollo profesional (IB y no IB) } \\
\text {-Promover el aprendizaje profesional a nivel individual, grupal e } \\
\text { institucional y hacer del aprendizaje profesional una parte integral de } \\
\text { la cultura escolar } \\
\text {-Organizar y desarrollar actividades de crecimiento profesional: } \\
\text { - Caminatas de aprendizaje } \\
\text { - Visitas pedagógicas al aula entre docentes } \\
\text { - Observación de pares } \\
\text { - Mentoría } \\
\text { - Participación en comunidades de aprendizaje } \\
\text { - Círculos de estudio: temática relacionada con la práctica } \\
\text { educativa } \\
\text { - Intercambio de experiencias y buenas prácticas entre docentes del } \\
\text { colegio o con docentes de otros centros } \\
\text { - Participación en proyectos de investigación-acción } \\
\text { - Autoformación } \\
\text { - Jornadas de formación para ampliar o profundizar en } \\
\text { conocimientos específicos }\end{array}$ \\
\hline $\begin{array}{l}\text { Planificar las necesidades } \\
\text { del centro a medio plazo } \\
\text { en especial para aquellos } \\
\text { puestos y competencias } \\
\text { claves en la estrategia del } \\
\text { colegio en los próximos } \\
\text { años }\end{array}$ & $\begin{array}{l}\text {-Identificar y clasificar el talento en el centro educativo con el } \\
\text { objetivo de ofrecer oportunidades de crecimiento personal y } \\
\text { profesional a los docentes en función de las necesidades del centro }\end{array}$ \\
\hline $\begin{array}{l}\text { Potenciar la participación } \\
\text { de los docentes en la red } \\
\text { de educadores del IB }\end{array}$ & $\begin{array}{l}\text {-Dar a conocer los puestos y roles disponibles en la red de } \\
\text { educadores del IB } \\
\text {-Facilitar la participación en actividades de la red de educadores del } \\
\text { IB }\end{array}$ \\
\hline
\end{tabular}

Fuente: Elaboración propia 
Tabla 10: Desempeño docente

Objetivo general: Mejorar el desempeño docente para apoyar la implementación del PD

Objetivo Específico 4:

Favorecer la integración y la comprensión del PD y sus requerimientos por parte de profesorado nuevo en el centro

\begin{tabular}{|c|c|}
\hline Actuaciones & Acciones \\
\hline $\begin{array}{l}\text { Desarrollar un plan de } \\
\text { acogida y formación para } \\
\text { el profesorado nuevo en } \\
\text { el centro }\end{array}$ & $\begin{array}{l}\text {-Planificar y llevar a cabo un programa de bienvenida y capacitación } \\
\text { inicial interna para docentes nuevos en el colegio que incluya lo } \\
\text { siguiente: } \\
\text { - Introducción a las normas para la implementación y } \\
\text { aplicaciones concretas del PD } \\
\text { - Introducción a la naturaleza del PD y las políticas asociadas } \\
\text { al programa } \\
\text { - Introducción al documento del PD: De los principios a la } \\
\text { práctica } \\
\text { - Introducción a la asignatura que se vaya a impartir del PD } \\
\text {-Introducción a los recursos y plataformas asociadas al PD (Mi IB, } \\
\text { Centro Recursos de los Programas, Comunidades de los } \\
\text { Programas...) } \\
\text {-Presentar comunidades de aprendizaje relevantes y posibilidades de } \\
\text { desarrollo profesional a partir de la colaboración con otros colegas }\end{array}$ \\
\hline $\begin{array}{l}\text { Proporcionar apoyo y } \\
\text { asistencia pedagógica de } \\
\text { forma regular para } \\
\text { docentes nuevos en el } \\
\text { centro }\end{array}$ & $\begin{array}{l}\text {-Ofrecer capacitación interna periódicamente y de forma } \\
\text { estructurada } \\
\text {-Hacer un seguimiento individual de docentes nuevos en el centro } \\
\text { para detectar áreas de desarrollo y determinar medidas de apoyo en } \\
\text { caso de necesidad }\end{array}$ \\
\hline $\begin{array}{l}\text { Facilitar y } \text { coordinar el } \\
\text { desarrollo profesional } \\
\text { del profesorado nuevo } \\
\text { en el centro }\end{array}$ & $\begin{array}{l}\text {-Asegurar la realización de talleres oficiales del PD a la primera } \\
\text { oportunidad disponible } \\
\text {-Disponer desarrollo profesional oficial adecuado a cada caso }\end{array}$ \\
\hline
\end{tabular}

Fuente: Elaboración propia

\section{CONCLUSIÓN}

Como se ha podido comprobar con la propuesta de actuaciones, las tres áreas de mejora determinadas están interconectadas y promueven un verdadero progreso en la implementación del Programa del Diploma, pero también abogan por una enseñanza de calidad en todo el colegio con la aspiración de la excelencia educativa. De esta forma, las tres áreas de mejora establecidas (Rendimiento académico del alumnado, Liderazgo pedagógico, Desempeño docente) se retroalimentan y apoyan mutuamente ya que son la base de todo plan de mejora de la eficacia escolar 
En este sentido, los colegios que ofrecen cualquiera de los programas del Bachillerato Internacional tienen la responsabilidad de establecer procedimientos para evaluar la eficacia de los programas y garantizar que se implementen adecuadamente en todo el colegio, con lo que esta propuesta de mejora se ajustaría perfectamente al requerimiento y a lo señalado en la literatura de la organización, en concreto en el documento Cómo hacer realidad el PEP. Liderazgo pedagógico en los colegios (p.21):

Los Colegios del Mundo del IB trabajan para cumplir con las normas de implementación establecidas por el IB, y ponen en práctica las aplicaciones concretas a fin de generar los cambios necesarios para mejorar la enseñanza y el aprendizaje y para fortalecer la comunidad y la cultura escolar. Se comprometen a fomentar la reflexión, mejorar las prácticas docentes y generar cambios duraderos a largo plazo.

La aspiración de este artículo es contribuir activamente a la mejora de la implementación del Programa del Diploma del Bachillerato Internacional en cualquier Colegio del Mundo IB. La pretensión es influir positivamente para iniciar un proceso de cambio en las áreas de desarrollo expuestas e incidir en la calidad educativa del colegio correspondiente. Lógicamente, como medida principal de seguimiento y evaluación de las acciones de mejora señaladas es necesario la inclusión de las actuaciones especificadas en el plan de acción/estratégico del colegio con el objetivo de darle relevancia y permitir la implicación de toda la comunidad escolar.

Este plan de mejora, indudablemente, debe ser flexible y estar abierto a las rectificaciones que sean necesarias, siendo esencial monitorizar de forma cercana la progresión y, sobre todo, el cumplimiento de los objetivos específicos establecidos. Para ello, es importante que regularmente se evalúe el nivel de cumplimiento de las actuaciones definidas con la finalidad de hacer las rectificaciones y modificaciones que sean necesarias de cara a los objetivos finales. Así, y en este sentido, es importante que el plan de mejora se vaya actualizando en función de necesidades detectadas y se vayan determinando nuevas acciones a medida que se evalúa en el tiempo. De esta forma, se recomienda también la inclusión en el plan de acción del colegio de una serie de indicadores de seguimiento de las distintas actuaciones que se proponen para facilitar la consecución de los objetivos marcados.

Por otra parte, también será necesario marcar una serie de indicadores de logro para permitir valorar el grado de consecución de cada uno de los objetivos al final del periodo de aplicación para valorar el grado de consecución. Sin embargo, es inevitable que el resultado en algunos casos sea difícil de cuantificar y, en estos casos, será necesario recurrir a evidencias de distinto tipo para determinar si el objetivo inicial propuesto se ha cumplido, o hasta qué punto se ha cumplido. Estos indicadores de logro deben ser establecidos en cada área de mejora determinada con la finalidad de obtener información con la que poder valorar el nivel de logro de los objetivos.

Estaríamos hablando así de una autoevaluación por parte del colegio que permitiría medir los resultados obtenidos y, a partir de la determinación del nivel de logro, proponer nuevas acciones de cara al futuro. Este proceso debería ser recurrente y cíclico ya que, partiendo del plan de mejora que se propone y su implementación posterior, el colegio debería hacer un ejercicio de reflexión y autoevaluación y determinar hasta qué punto ha alcanzado los resultados que esperaba y, en función de estos, establecer una nueva propuesta de mejora para los siguientes años.

No obstante, esta evaluación del proyecto debería contar con otros instrumentos de recogida de información, como cuestionarios a distintos miembros de la comunidad escolar, entrevistas, reuniones y seminarios, grupos de discusión, etc, que apoyaría la reflexión sobre el nivel de consecución. 
Además, y como parte del proceso relacionado con el plan de mejora, los programas del Bachillerato Internacional están sometidos a evaluaciones quinquenales regulares. De esta forma, además de la autoevaluación que realice el centro educativo, también se contará con una evaluación por parte de una organización externa, como es el IB, cuando se lleve a cabo la evaluación quinquenal correspondiente.

Sin embargo, y aunque el plan de mejora tenga un seguimiento y una evaluación continua, la idea principal es establecer una cultura de progreso constante en el centro educativo y en toda su comunidad escolar. Así, Murillo y Krichesky (2012) remarcan esta idea como aspecto central:

La mejora de la escuela no puede ser considerada una experiencia aislada o puntual que adjetive a unos pocos centros en momentos específicos de su historia. La voluntad de optimizar la calidad educativa ha de constituirse como una señal de identidad permanente de todas y cada una de las escuelas. (p.27).

Por lo tanto, el objetivo final es establecer un proceso de mejora que sea sostenible en el tiempo y que permita un progreso constante del colegio y de sus prácticas de enseñanza y aprendizaje, y no solo en las áreas señaladas. Es decir, en definitiva, se trata de consolidar una cultura de superación permanente que permita hacer avanzar los programas educativos, evitando instalar la idea de la mejora como algo puntual que se lleva a cabo cuando se han detectado dificultades ocasionales o el colegio debe someterse a una evaluación externa. 


\section{BIBLIOGRAFÍA}

Ávalos, B. (2011). El liderazgo docente en comunidades de práctica. Educar, 47(2), 237-252.

Bolívar, A. (2007) Cómo mejorar los centros educativos. Capítulos 1 y 2 (pp. 15-53) Madrid: Síntesis.

Bolívar, A., López-Yáñez, J. y Murillo, F.J. (2013) Liderazgo en las instituciones educativas. Una revisión de líneas de investigación. Revista Fuentes, 14, 15-60.

Gairín, J. y Rodríguez-Gómez, D. (2011) Cambio y mejora en las organizaciones educativas. Educar, 47(1) 31-50.

Gairín, J. (1998). Los estadios de desarrollo organizacional. Contextos educativos. Revista de educación. (1), 125-154

Gento Palacios, S. (1998). Marco referencial para la evaluación de un proyecto educativo. Educación XXI, 1, 93.

Gento Palacios, S. (2012). Evaluación de la calidad de las instituciones educativas. Granada: CIOIE.

Guarro, A. (2013) ¿Cómo ayudar a los centros escolares a 'moverse’? Autonomía y evaluación. III Congreso Nacional de Equipos Directivos de Centros: "Evaluación, cambio y mejora". CEFIRE, Valencia, 14-16 de noviembre.

Guarro, A. (2005). Los procesos de cambio educativo en una sociedad compleja. Diseño, desarrollo e innovación del currículum. Madrid: Psicología Pirámide.

Hargreaves, A. y Fink, D. (2006) Estrategias de cambio y mejora en educación caracterizadas por su relevancia, difusión y continuidad en el tiempo. Revista de Educación, 339, 43-58.

Ley Orgánica 2/2006, de 3 de mayo, de Educación, modificada por Ley Orgánica 8/2013, de 9 de diciembre, para la mejora de la calidad educativa.

López-Yáñez, J. y Sánchez-Moreno, M. (2000) Acerca del cambio en los sistemas complejos. En Estebaranz, A. Construyendo el cambio: perspectivas y propuestas de innovación educativa.

Sevilla: Servicio de Publicaciones de la Universidad de Sevilla.

Murillo Torrecilla, F. (2006). Una dirección escolar para el cambio: del liderazgo transformacional al liderazgo distribuido. REICE - Revista Electrónica Iberoamericana sobre Calidad, Eficacia y Cambio en Educación, 4(4e). 1-24.

Murillo, F. J., \& Krichesky, G. J. (2012). El proceso del cambio escolar. Una guía para impulsar y sostener la mejora de las escuelas. REICE. Revista Iberoamericana sobre Calidad, Eficacia y Cambio en Educación, 10(1).

Murillo, F.J., Martínez-Garrido, C.A. y Hernández, R. (2011) Decálogo para una enseñanza eficaz. REICE. Revista Iberoamericana sobre Calidad, Eficacia y Cambio en Educación, 9(1) 627. 
Organización del Bachillerato Internacional. (2014). Normas para la implementación de los programas y aplicaciones concretas. Cardiff. UK.

Organización del Bachillerato Internacional. (2017). Qué es la educación IB. Cardiff. UK.

Organización del Bachillerato Internacional. (2008). Hacia un continuo de programas de educación Internacional. Cardiff: UK.

Organización del Bachillerato Internacional. (2019). Programa de la Escuela Primaria. Cardiff. UK.

Organización del Bachillerato Internacional. (2007) Cómo hacer realidad el PEP. Liderazgo pedagógico en los colegios. Cardiff. UK.

Organización del Bachillerato Internacional. (2019). Programa de los Años Intermedios. Cardiff. UK.

Organización del Bachillerato Internacional. (2014). El Programa de los Años Intermedios: de los principios a la práctica. Cardiff. UK.

Organización del Bachillerato Internacional. (2019). Programa del Diploma. Cardiff. UK.

Organización del Bachillerato Internacional. (2015). El Programa del Diploma: de los principios a la práctica. Cardiff. UK.

Organización del Bachillerato Internacional. (2015) El Programa de Orientación Profesional: de los principios a la práctica. Cardiff. UK.

Organización del Bachillerato Internacional. (2019). Programa de Orientación Profesional. Cardiff. UK.

Organización del Bachillerato Internacional. (2015). Guía para la evaluación de los programas. Cardiff. UK.

Organización del Bachillerato Internacional. (2019). The IB Middle Years Programme Final Statistical Bulletin May 2019 Examination Session. Cardiff. UK.

Organización del Bachillerato Internacional. (2019). The IB Diploma Programme Final Statistical Bulletin May 2019 Examination Session. Cardiff. UK.

Santos Guerra, M. (2000). La escuela que aprende. Barcelona: Ediciones Morata.

Senge, P. (1990). La quinta disciplina. El arte y la práctica de la organización abierta al aprendizaje. Barcelona: Granica. 


\section{SOBRE EL AUTOR}

\section{Antonio Muñoz Castillo}

Antonio Muñoz trabaja en el Centro Global del IB en La Haya desde hace 4 años, en el departamento de Colegios del Mundo IB. Antonio es responsable de un porfolio de colegios públicos y privados en España y América Latina (Argentina, Chile, Perú, Costa Rica, México y Colombia), a los cuales brinda apoyo y orientación en relación con la implementación de los programas IB.

Su trabajo en el departamento de Colegios del Mundo IB incluye también gestionar las relaciones con los Colegios del mundo IB, grupos de Colegios del Mundo del IB, Ministerios de Educación y asociaciones de Colegios del Mundo del IB y establecer un apoyo proactivo para los colegios con el fin de optimizar la implementación de los programas del IB.

Antes de vincularse profesionalmente con la organización del Bachillerato Internacional, trabajó en escuelas internacionales durante más de 20 años desempeñando cargos de liderazgo pedagógico e impartiendo docencia, tanto en el PAI como en el PD. Además, ha desempeñado múltiples roles como educador de la red de educadores del IB (IBEN). Es licenciado en Filología Hispánica por la Universidad Complutense y tiene una maestría en Dirección, Innovación y Liderazgo de Centros Educativos por la Universidad Camilo José Cela de Madrid y un posgrado de Experto en innovación, metodología docente y evaluación aplicadas a la educación por la Universidad La Salle de Madrid.

\section{Contact information:}

Antonio Muñoz Castillo, IB World Schools Manager

International Baccalaureate Organization (IB Global Centre, The Hague)

Churchillplein 6, $2517 \mathrm{JW}$

La Haya,

Países Bajos

antonio.munoz@ibo.org 